\title{
XIAP inhibits mature Smac-induced apoptosis by degrading it through ubiquitination in NSCLC
}

\author{
SIDA QIN $^{1 *}$, CHENGCHENG YANG $^{2 *}$, BOXIANG ZHANG $^{1}$, XIANG LI $^{1}$, XIN SUN $^{1}$, GANG LI $^{1}$, \\ JING ZHANG ${ }^{1}$, GUODONG XIAO ${ }^{1}$, XIAO GAO $^{1}$, GUANGHONG HUANG ${ }^{1}$, PEILI WANG ${ }^{1}$ and HONG REN ${ }^{1}$ \\ ${ }^{1}$ Department II of Thoracic Surgery, The First Affiliated Hospital of Xi'an Jiaotong University, Xi'an, Shaanxi 710061; \\ ${ }^{2}$ Department of Oncology, The First Affiliated Hospital of Xi'an Jiaotong University, Xi'an, Shaanxi 710061, P.R. China
}

Received May 13, 2016; Accepted June 25, 2016

DOI: $10.3892 /$ ijo.2016.3634

\begin{abstract}
X-linked inhibitor of apoptosis protein (XIAP) and second mitochondrial-derived activator of caspase (Smac) are two important prognostic biomarkers for cancer. They are negatively correlated in many types of cancer. However, their relationship is still unknown in lung cancer. In the present study, we found that there was a negative correlation between Smac and XIAP at the level of protein but not mRNA in NSCLC patients. However, XIAP overexpression had no effect on degrading endogenous Smac in lung cancer cell lines. Therefore, we constructed plasmids with full length of Smac (fSmac) and mature Smac (mSmac) which located in cytoplasm instead of original mitochondrial location, and was confirmed by immunofluorescence. Subsequently, we found that mSmac rather than fSmac was degraded by XIAP and inhibited cell viability. CHX chase assay and ubiquitin assay were performed to illustrate XIAP degraded mSmac through ubiquitin pathway. Overexpression of XIAP partially reverted apoptotic induction and cell viability inhibition by mSmac, which was due to inhibiting caspase-3 activation. In nude mouse xenograft experiments, mSmac inhibited Ki-67 expression and slowed down lung cancer growth, while XIAP partially reversed the effect of mSmac by degrading it. In conclusion, XIAP inhibits mature Smac-induced apoptosis by degrading it through ubiquitination in NSCLC.
\end{abstract}

\section{Introduction}

Apoptosis is the process of programmed cell death that includes many biochemical changes, such as nuclear fragmentation,

Correspondence to: Dr Hong Ren, Department II of Thoracic Surgery, The First Affiliated Hospital of Xi'an Jiaotong University, Hong Ren, 277 West Yanta Road, Xi'an, Shaanxi 710061, P.R. China E-mail: renhong@xjtu.edu.cn

${ }^{*}$ Contributed equally

Key words: X-linked inhibitor of apoptosis protein, Smac, apoptosis, ubiquitin, non-small cell lung cancer cell shrinkage and chromatin condensation. It plays a crucial role in the growth, development, metabolism and maintaining homeostasis in multicellular organisms (1). If some internal or external factors effect the production of apoptosis, a disruption of the balance between cell proliferation and apoptosis would lead to the development of cancer (2). Inhibitor of apoptosis protein (IAP) is one of the best known factors which can promote cancer development by inhibiting cell apoptosis (3).

As a member of IAP family, X-linked inhibitor of apoptosis protein (XIAP) contains three baculoviral IAP repeat (BIR) domains and one really interesting new gene (RING)finger domain (4). The BIR1, BIR2 and BIR3 domains of XIAP are the main structure for inhibiting apoptosis, due to their capability of inhibiting caspases. The BIR 2 domain plays an important role in blocking caspase- 3 and -7 , and the BIR3 domain is required for inhibition of caspase-9 (5). Like other IAP members, XIAP contains RING domain in its C terminal, which is relative to its self-ubquitination. Besides, its RING domain also promotes ubquitination of other proteins and degrades them through ubiquitin-proteasome pathway (6).

Second mitochondrial-derived activator of caspase (Smac) is located in mitochondria. It releases into cytosol under some apoptotic induction signals, such as chemotherapy or radiotherapy (7). Its main mechanism of apoptotic induction is the interaction between Smac and IAPs. Smac binds to IAPs and reverses the inhibition of caspases by IAPs, and then induces the caspase cascade and cell apoptosis (8). For example, Smac binds to the BIR domains of XIAP through its N-terminus and prevents XIAP from binding caspase-3, -7 and -9 , then, the caspases were activated and amplified to trigger cell apoptosis (9).

XIAP and Smac are two important prognostic biomarkers for malignant tumors because they determine the sensitivity of cancer cells to apoptosis (10-14). Usually, the expression of XIAP is much higher in cancer tissues than that in normal tissues, and the increased XIAP expression is correlated with poor prognostic and decreased survival in patients (15-17). While Smac expression was reduced in cancer tissues compared to normal tissues, and this reduction is more significant during cancer progression with advanced malignant phenotypes $(18,19)$. It has been reported that the expression of XIAP and Smac are negatively correlated in many types of cancer, such as testicular germ cell tumors, renal cell 
carcinomas and gastric adenocarcinomas (19-21). However, its mechanisms are still unknown.

In the present study, we found that there was a negative correlation between Smac and XIAP at the level of protein but not mRNA in NSCLC patients. Overexpression of mature Smac induced lung cancer cells apoptosis, and this apoptotic induction was inhibited by co-transfection with XIAP plasmid. It is because XIAP promoted the ubiquitination and degradation of Smac. Besides, nude mouse xenograft experiment further proved the relation and the function of Smac and $\mathrm{XIAP}$ in vivo.

\section{Materials and methods}

Clinical samples. Primary tumor specimens were obtained from 26 patients with NSCLC who underwent pulmonary surgery at the First Affiliated Hospital of Xi'an Jiaotong University. Normal lung tissues were collected at $>5 \mathrm{~cm}$ from the edge of the tumor. All patients had a single tumor, without distant metastasis and none of them had been previously treated with chemo- or radiotherapy. The study design and procedure were approved by the Ethics Committee of the hospital and each participant signed an informed consent document prior to enrollment.

$R T-P C R$. Total RNA was extracted from tissues using RNeasy Mini kits (Qiagen, Inc., Valencia, CA, USA) and reverse transcription was performed with SuperScript III First-Strand kits (Invitrogen Life Technologies, Carlsbad, CA, USA) according to the manufacturer's instructions. The DNA was amplified with PfuUltra DNA Polymerases \& PCR Master Mixes (Agilent Technologies, Palo Alto, CA, USA) on an S1000 ${ }^{\mathrm{TM}}$ Thermal Cycler (Bio-Rad Laboratores, Richmond, CA, USA), with the following protocol: initial denaturation at $95^{\circ} \mathrm{C}$ for $3 \mathrm{~min}$, then 30 cycles of $95^{\circ} \mathrm{C}$ for $30 \mathrm{sec}, 60^{\circ} \mathrm{C}$ for $30 \mathrm{sec}$, and $72^{\circ} \mathrm{C}$ for $1 \mathrm{~min}$, and then final extension at $72^{\circ} \mathrm{C}$ for $5 \mathrm{~min}$. GAPDH gene was used as a control. The following PCR primer sequences were used for Smac forward, 5'-ATCAGAG CCTCATTCCCTTAGT-3' and reverse, 5'-TGGTGTTTTGA AGTCATCTCAGC-3'; XIAP forward, 5'-TGCTCACCTAAC CCCAAGAG-3' and reverse, 5'-ATCTGCCATGGATGGA TTTC-3'; GAPDH forward, 5'-CACCAACTGCTTAGCAC CCC-3' and reverse, 5'-TGCTCAGTGTAGCCCAGGATG-3'. The RT-PCR assay was analyzed by grey value analysis using Quantity One software; the target/internal relative grey-scale value indicates the corresponding mRNA expression level.

Western blot analysis and co-immunoprecipitation assay. Lung tissues or treated cells were lysed in protein extraction buffer [50 mM Tris-Cl (pH 7.5), $150 \mathrm{mM} \mathrm{NaCl,} 1$ mM EDTA (pH 8.0), 1\% Triton X-100, and protease inhibitor cocktail (Roche Molecular Biochemicals, Indianapolis, IN, USA)] for $30 \mathrm{~min}$ on ice. Western blot analysis and co-immunoprecipitation assay were performed as previously described (22) using the following antibodies: anti-Smac rabbit monoclonal antibody (1:1,000; ab32023), anti-XIAP rabbit polyclonal antibody $(1: 2,000 ;$ ab21278), anti- $\beta$-actin rabbit polyclonal antibody (1:5,000; ab75186) (both from Abcam, Cambridge, $\mathrm{UK})$; anti-GAPDH rabbit polyclonal $\mathrm{IgG}$ antibody $(1: 1,000$, sc-25778; Santa Cruz Biotechnology, Inc., Santa Cruz, CA,
USA); anti-Flag tag mouse monoclonal IgG antibody (1:1,000, F1804; Sigma-Aldrich, St. Louis, MO, USA); anti-myc tag mouse monoclonal antibody $(1: 1000 ; 2276 S$; Cell Signaling Technology, Inc., Danvers, MA, USA); anti-ubiquitin rabbit monoclonal antibody (1:2000; ab140601; Abcam).

Construction of plasmids and transfection. Full-length XIAP and Smac were generated by PCR from A549 cell cDNA using the following primers: XIAP: forward 5'-AGATCTGGATC CACTTTTAACAGTTTTGAAGG-3' and reverse 5'-TCTAGA GCGGCCGCTTAAGACATAAAAATTTTTTGCTTG-3'. Smac: sense 5'-AAGCTTGGATCCGCGGCTCTGAAGAGT TGGCTGTCG-3' and reverse 5'-TCTAGAGCGGCCGCTCA ATCCTCACGCAGGTAGGCCTCC-3'; Ub-mSmac was generated by overlapping PCR using primiers: forward 5'-AGATCTGGATCCATGCAGATCTTCGTGAAAACCCT TACCGGC-3' and reverse 5'-TCTAGAGCGGCCGCTCAA TCCTCACGCAGGTAGGCCTCC-3'. The resulting fragments were digested by BamHI and NotI (Thermo Fisher Scientific, Rockford, IL, USA), and cloned into pcDNA3.0Flag. The plasmids were transfected into A549 or H460 cells with Lipofectamine 2000 (Invitrogen-Life Technologies). Western blotting was used to identify protein overexpression in cells.

MTT assay. For cell viability assays, $5 \times 10^{3}$ A549 and H460 cells, with or without overexpression of Smac or mSmac, were plated into each well of 96-well plates. After $48 \mathrm{~h}, 20 \mu \mathrm{l}$ of MTT (Sigma-Aldrich) was added to each well for $4 \mathrm{~h}$. Then, the supernatant was discarded and $100 \mu \mathrm{l}$ DMSO was added to each well, and the absorbance at $490 \mathrm{~nm}$ (A490) was measured with a microplate reader (Bio-Rad Laboratories). Each cell viability assay was performed with five replicate wells. Cell viability (\%) was calculated as follows: Cell viability $=$ the average A490 in an experimental chemotherapy group/the average A490 in the blank control group x $100 \%$.

Flow cytometry. Cells were plated into 24-well plates with a concentration of $1 \times 10^{5} /$ well. The following day, cells were transfected with mSmac and/or XIAP. After 48 h, cells were trypsinized and incubated with $5 \mu \mathrm{l}$ propidium iodide and $5 \mu \mathrm{l}$ Annexin V-FITC (Invitrogen) for $15 \mathrm{~min}$. Samples were then analyzed for apoptosis by a FACScan flow cytometer (BD Biosciences, Franklin Lakes, NJ, USA).

Immunofluorescence. Lung cancer cells were fixed with $3 \%$ paraformaldehyde and permeabilized using $0.2 \%$ Triton X-100. Then the fixed cells were incubated with anti-Flag tag mouse monoclonal IgG antibody (1:1,000, F1804; Sigma-Aldrich). The secondary antibody is a labeled goat anti-mouse IgG antibody (A11001; Invitrogen, Carlsbad, CA, USA). Fluorescence confocal images were captured using a LSM 5 Pascal Laser Scanning Microscope (Carl Zeiss AG, Oberkochen, Germany) and Laser Scanning Microscope LSM PASCAL software (version 4.2 SP1).

Caspase-3 activity assay. Cells were washed with cold PBS twice, resuspended in lysis buffer and kept on ice for $15 \mathrm{~min}$. The lysate was centrifuged at $16,000 \mathrm{x} \mathrm{g}$ at $4^{\circ} \mathrm{C}$ for $15 \mathrm{~min}$. Supernatants were collected and caspase- 3 activity 

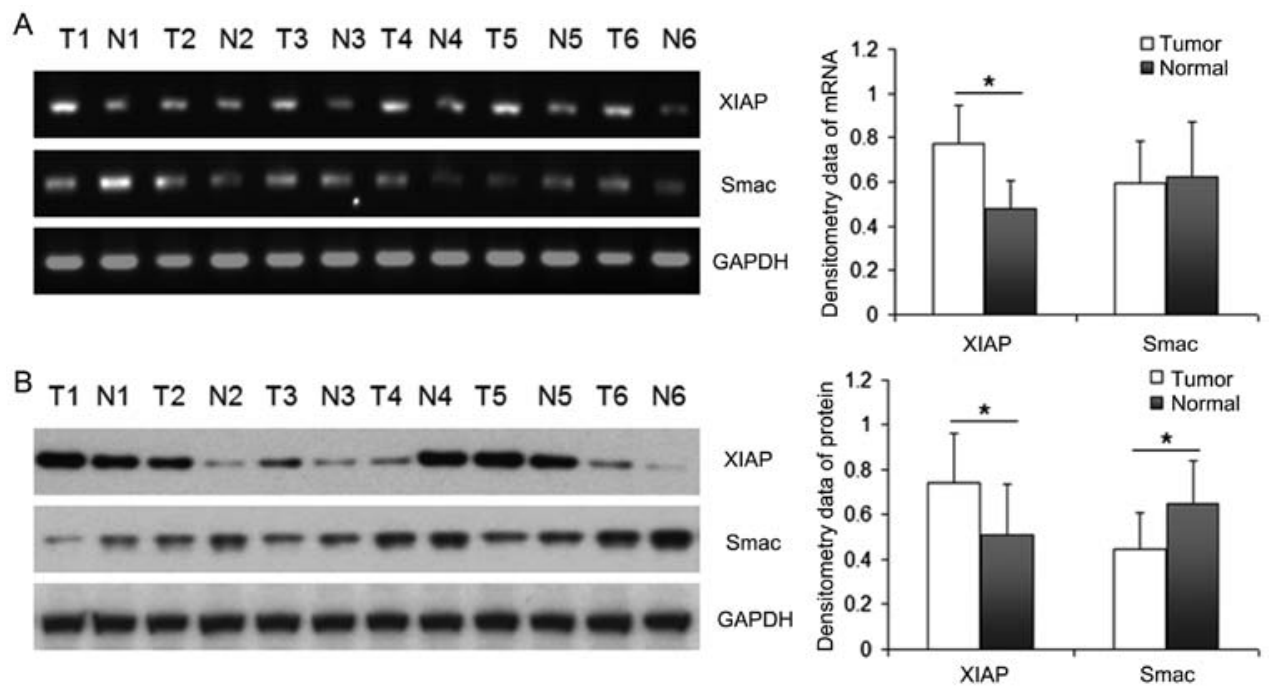

Figure 1. XIAP and Smac in NSCLC tumor tissues and matched lung tissues. (A) RT-PCR and (B) western blot analysis of Smac and XIAP in cancer (T) and matched para-tumor tissues $(\mathrm{N})$ are shown. The bands were quantified by densitometry and normalized to GAPDH. Values are depicted as mean \pm SD, $\mathrm{P}<0.05$.

was measured using caspase-3 activity assay kit (Beyotime Institute of Biotechnology, Shanghai, China) according to the manufacturer's instructions. The working principles of this kit are based on the cleavage of the caspase-3 substrate, Ac-DEVD-pNA. The release of p-nitroanilide (pNA) was qualified by determining the absorbance with the microplate reader at $405 \mathrm{~nm}$. Caspase- 3 activity was expressed as the ratio of control cells.

Nude mouse xenograft studies. Four-week-old BALB/c athymic nude mice (4-5-weeks old and weighing 16-20 g) were purchased from the Center of Laboratory Animals of Xi'an Jiaotong University and were bred at pathogen-free conditions. All animal experiments were approved by the Ethics Committee of the First Affiliated Hospital of Xi'an Jiaotong University. H460 cells were transfected with empty vector, Ub-mSmac or Ub-mSmac+XIAP, and stably-transfected cells were selected in medium supplemented with $1 \mathrm{mg} / \mathrm{ml} \mathrm{G} 418$ (Sigma-Aldrich) for one month. Western blotting was used to identify the cells with significant overexpression. Cells $\left(5 \times 10^{6}\right)$ were harvested from tissue culture dishes and subcutaneously injected into the right flank of each nude mice. Tumor volumes were measured every three days by calipers and calculated using the formula: Tumor volume $=$ length $\mathrm{x}$ width ${ }^{2} \mathrm{x} 1 / 2$. After one month, the mice were sacrificed and the tumors were removed for IHC staining.

IHC staining. The expression of XIAP, Smac and Ki-67 was evaluated by immunohistochemical (IHC) analyses in $4-\mu \mathrm{m}$ sections of the $10 \%$ formaldehyde-fixed and paraffinembedded blocks. The tissue slides were deparaffinized in xylene and rehydrated through a series of graded alcohols. To exhaust endogenous peroxidase activity, the slides were treated with $3 \% \mathrm{H}_{2} \mathrm{O}_{2}$ for $10 \mathrm{~min}$. Then, the slides were immersed in $0.01 \mathrm{M}$ citrate buffer, $\mathrm{pH} 6.0$, for $3 \mathrm{~min}$ in a pressure cooker at $125^{\circ} \mathrm{C}$, then placed at room temperature for $30 \mathrm{~min}$ to cool down. After washing with phosphate-buffered saline (PBS), the slides were pre-incubated for $30 \mathrm{~min}$ in $10 \%$ normal goat serum and then incubated with anti-Smac rabbit monoclonal antibody (1:400; ab32023), anti-XIAP rabbit polyclonal antibody (1:400; ab21278; both from Abcam); anti-Ki-67 rabbit monoclonal antibody (1:400, 9027; Cell Signaling Technology). After washing with PBS, the slides were incubated in a donkey anti-rabbit IgG/HRP secondary antibody (1:250; bs-0295D-HRP; Beijing Bioss, Inc., Beijing, China) at room temperature for $30 \mathrm{~min}$. All slides were generated using a diaminobenzidine chromogen solution. The counterstaining was performed with hematoxylin. The slides then were dehydrated, cleared and mounted.

Both the intensity and extent of staining were taken into consideration when analyzing the data. The extent of staining was scored from 0 to $100 \%(1,1-25 \%, 2,26-50 \%, 3,51-75 \%, 4$, $76-100 \%)$ and the intensity of staining was scored from 0 to 2 ( 0 , none; 1 , weak to moderate; 2 , strong). The IHC score was determined as high expression $(+)$ : score $\geq 3$; low expression $(-)$ : score $\leq 2$.

Statistical analysis. Data reported are the mean \pm SD deviation of three independent experiments and evaluated by the Student t-tests or one-way ANOVA. The normal distribution and equality of variances of the data in clinical samples were detected by Kolmogorov-Smirnov test and Levene's test separately. The difference of XIAP or Smac expression between cancer tissues and their paired normal tissues was evaluated by paired-samples t-test. The correlation between the expression of XIAP and Smac was evaluated by Pearson's correlation analysis. Statistical analyses were performed using the SPSS version 13.0 (SPSS, Inc., Chicago, IL, USA). Significant differences were established at $\mathrm{P}<0.05$.

\section{Results}

Expression of XIAP and Smac in NSCLC. RT-PCR and western blotting were performed to detect the levels of mRNA and protein of Smac and XIAP in 26 NSCLC tissues and their matched para-tumor tissues. Representative result is 


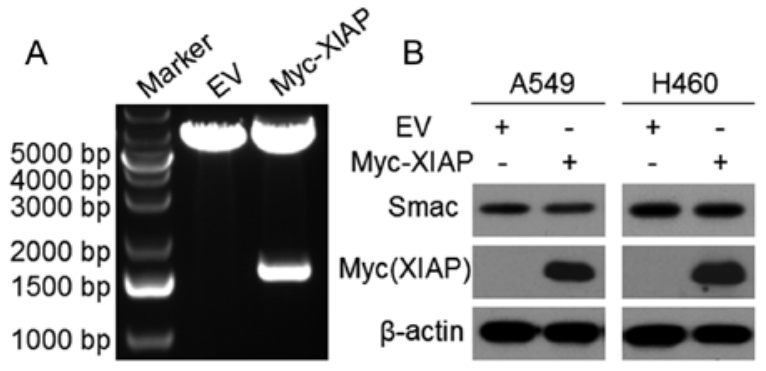

Figure 2. XIAP did not degrade endogenous Smac in lung cancer cell lines. (A) Myc-XIAP and empty vector (EV) were double digested by restriction enzymes BamHI and NotI. (B) The expression of XIAP and Smac were evaluated by western blotting in the A549 and $\mathrm{H} 460$ cells following transfection with Myc-XIAP or EV for $48 \mathrm{~h}$. $\beta$-actin was used as loading control.

shown in Fig. 1. The bands were quantified by densitometry and normalized to GAPDH. The data in different groups were normal distribution and equality of variances, so we used paired-samples t-test. The mRNA of XIAP in NSCLC tissues was significantly higher than that in the matched para-

A

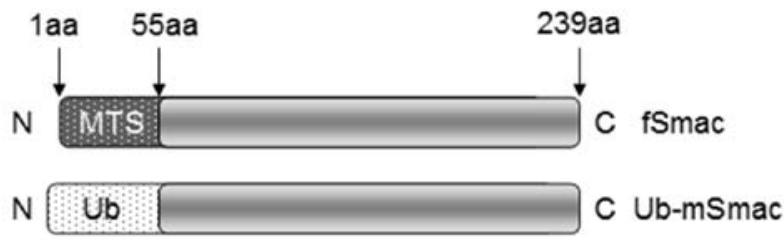

C
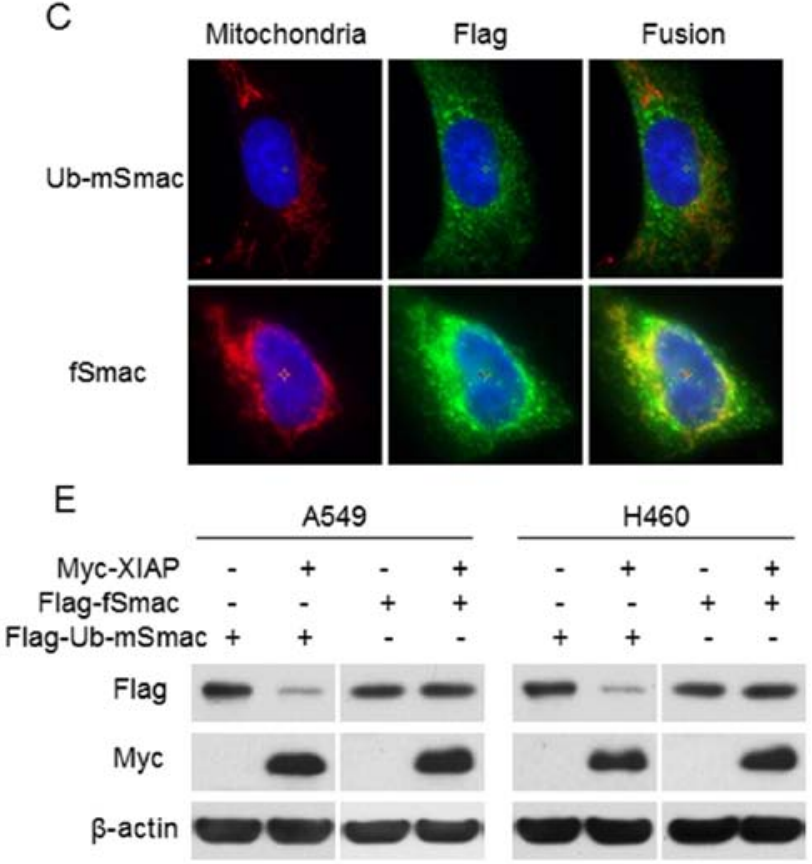

tumor tissues $(\mathrm{P}<0.05)$, while the mRNA of Smac was not significantly different between cancer tissues and para-tumor tissues. The protein expression of XIAP in NSCLC tissues was also significantly higher than that in the matched lung tissues $(\mathrm{P}<0.05)$, which corresponded with the mRNA level. However, the protein expression of Smac was significantly higher in para-tumor tissues than that in NSCLC tissues $(\mathrm{P}<0.05)$. There was a negative correlation between Smac and XIAP only on the level of protein $(r=-0.59, \mathrm{P}<0.05)$ but not on the level of mRNA in the cancer samples of NSCLC patients $(\mathrm{P}>0.05)$

XIAP could not degrade endogenous Smac in lung cancer cell lines. We constructed plasmid pcDNA3-Myc-XIAP and confirmed it by sequencing and double digestion (Fig. 2A). To investigate the effects of XIAP to endogenous Smac, we transfected Myc-XIAP or empty vector into A549 and H460 cells for $48 \mathrm{~h}$. Then, western blotting confirmed that the expression of XIAP in the XIAP-transfected cells was higher than that in the control cells. However, the expression of Smac did not change prominently after XIAP overexpression, thus, XIAP could not degrade endogenous Smac in NSCLC cells (Fig. 2B).

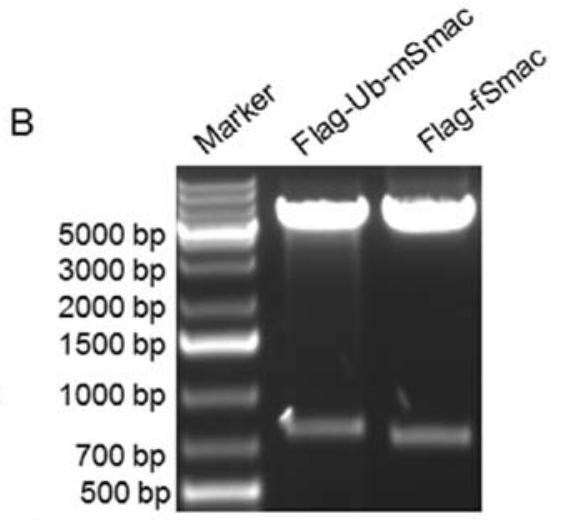

$\mathrm{D}$
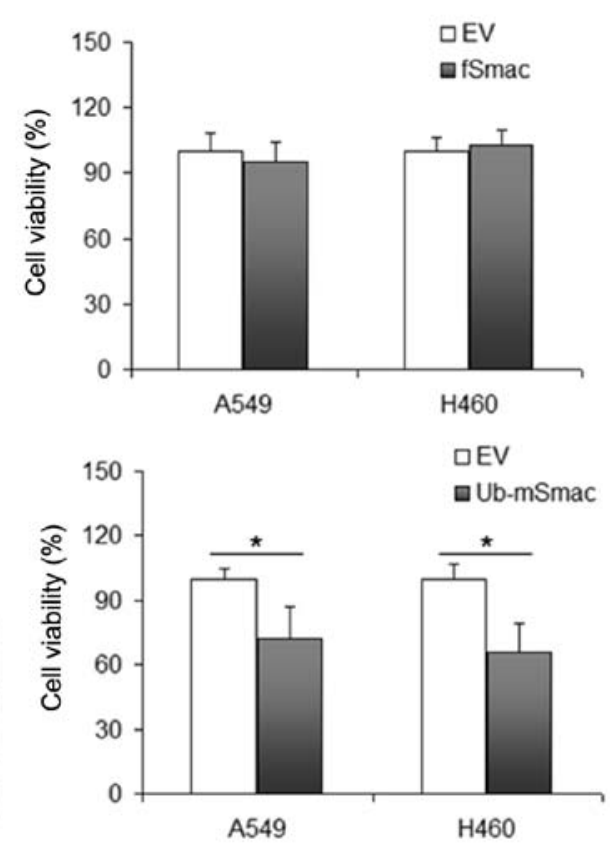

Figure 3. The location and function of fSmac and mSmac. (A) The structure of fSmac and Ub-mature Smac fusion protein (Ub-mSmac). (B) Flag-fSmac and Flag-Ub-mSmac were double digested by restriction enzymes BamHI and NotI. (C) The Localization of fSmac and Ub-mSmac were evaluated by immunofluorescence staining in the U2OS cells following transfection with Flag-fSmac or Flag-Ub-mSmac for $48 \mathrm{~h}$. (D) Cell viability was measured by MTT assay following transfected with fSmac or Ub-mSmac in A549 and H460 cells for 48 h. (E) Cells were transfected with Myc-XIAP and Flag-fSmac or Flag-Ub$\mathrm{mSmac}$ for $48 \mathrm{~h}$ and exogenous Smac and XIAP were evaluated by western blotting with anti-Flag or anti-Myc antibody. $\beta$-actin was used as loading control. 

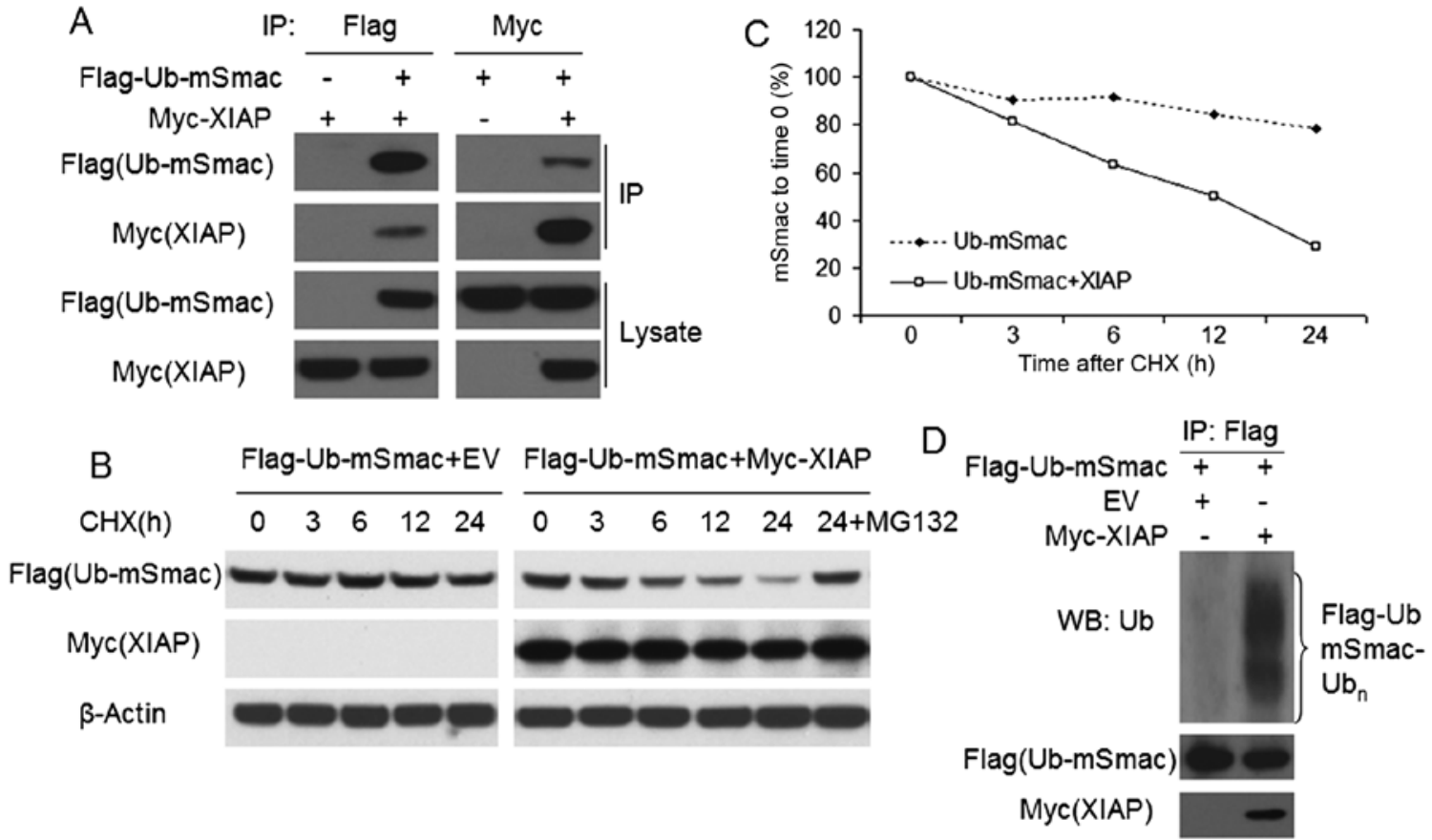

Figure 4. XIAP bound to mSmac and promoted the ubiquitin-mediated proteolysis of mSmac. (A) Flag-Ub-mSmac and Myc-XIAP plasmids were transfected into H460 cells. Ub-mSmac or XIAP was immunoprecipitated with anti-Flag or anti-Myc antibody. Mature Smac and XIAP coimmunoprecipitated with each other. (B) The half-life of mSmac was analyzed following treatment with cycloheximide (CHX). The degradation of mSmac was faster in XIAP overexpressing $\mathrm{H} 460$ cells, and treatment with MG132 inhibited XIAP induced mSmac degradation. (C) The intensity of mSmac was normalized to $\beta$-actin and depicted in a line chart. (D) Flag-Ub-mSmac was precipitated from Flag-Ub-mSmac overexpressing H460 cells by immunoprecipitation using an anti-Flag antibody. Smac ubiquitination was detected by western blotting.

The location and function of Smac and mature Smac. The first 55 amino acids at the $\mathrm{N}$ terminal of Smac constitute a mitochondrial targeting signal (MTS) peptide and are cleaved to generate mature Smac. We constructed plasmids pcDNA3Flag-fSmac and pcDNA3-Flag-Ub-mSmac. Their structures are shown in the Fig. $3 \mathrm{~A}$ and their digestion results were confirmed by sequencing and double digestion (Fig. 3B). To further identify the expression and the location of fSmac and mSmac, we transfected Flag-fSmac or Flag-Ub-mSmac into U2OS cells, which are commonly used to detect protein localization because their appropriate nucleus to cytoplasm ratio. By immunofluorescence, we found Flag-fSmac was mainly located in the mitochondria and Flag-Ub-mSmac was located in the cytoplasm (Fig. 3C).

After transfection with Flag-fSmac or Flag-Ub-mSmac into lung cancer A549 and H460 cells for 48 h, cell viability was assessed using an MTT assay. We found that $\mathrm{mSmac}$ but not fSmac inhibited cell viability (Fig. 3D). Cells were co-transfected with Myc-XIAP and Flag-fSmac or FlagUb-mSmac for $48 \mathrm{~h}$, and exogenous Smac and XIAP were evaluated by western blotting, which showed XIAP inhibited the expression of mSmac but not fSmac (Fig. 3E).

XIAP degrades mSmac through ubiquitin pathway. To study how XIAP degrades $\mathrm{mSmac}$ in NSCLC, we transfected Flag-Ub-mSmac and/or Myc-XIAP into H460 cells for $48 \mathrm{~h}$. Reciprocal co-immunoprecipitation was done by using anti-Flag or anti-Myc antibodies to confirm the interaction between XIAP and mSmac (Fig. 4A). Then, CHX chase assays was done by cell treatment with a protein synthesis inhibitor, cycloheximide (CHX), for $0-24 \mathrm{~h}$ to analyze the XIAP mediated downregulation of $\mathrm{mSmac}$ in Flag-UbmSmac with/without Myc-XIAP transfected H460 cells. In the course of time, the half-life of mSmac was prominently decreased in co-transfected cells, compared with that in the cells with mSmac transfection alone (Fig. 4B and C). Besides, the downregulation of mSmac was prevented by MG132, a proteasome inhibitor (Fig. 4B). Moreover, ubiquitin assay was done by western blotting using anti-ubiquitin antibody in cells transfected with Flag-Ub-mSmac with/without Myc-XIAP. As shown in Fig. 4D, XIAP overexpression substantially increased the ubiquitination of mSmac in H460 cells.

The function of XIAP and mSmac in vitro and in vivo. To explore the function of XIAP and $\mathrm{mSmac}$ in lung cancer cells, we transfected Flag-Ub-mSmac and/or Myc-XIAP into $\mathrm{H} 460$ cells for $48 \mathrm{~h}$. Western blotting was done to confirm that exogenous XIAP can degrade mSmac (Fig. 5A). Cell apoptosis and viability were evaluated by Annexin V/PI analysis and MTT assays separately. Mature Smac promoted apoptosis and inhibited cell viability in lung cancer H460 cells, while XIAP partially reverted the effect of exogenous mSmac, leading to a significantly decreased cell apoptosis and increased cell viability $(\mathrm{P}<0.05$, Fig. 5B-D). Furthermore, caspase assay was performed to show that $\mathrm{mSmac}$-induced apoptosis was due to the increased caspase- 3 activation, and the reversion of apoptosis by XIAP was caused by decreasing the activation of caspase-3 (Fig. 5E).

To further confirm this result in vivo, we stably transfected Flag-Ub-mSmac with/without Myc-XIAP into H460 cells 
A

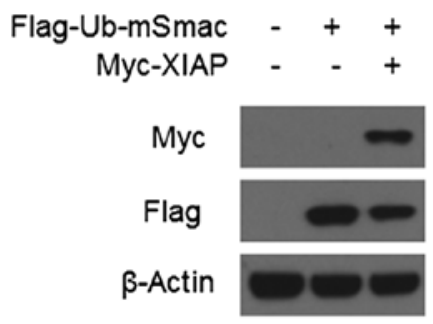

C

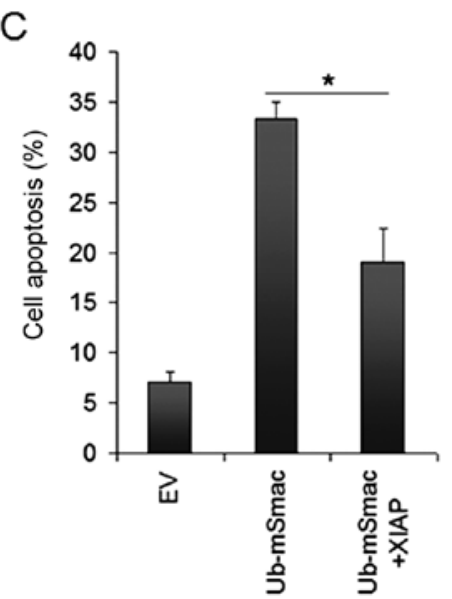

B

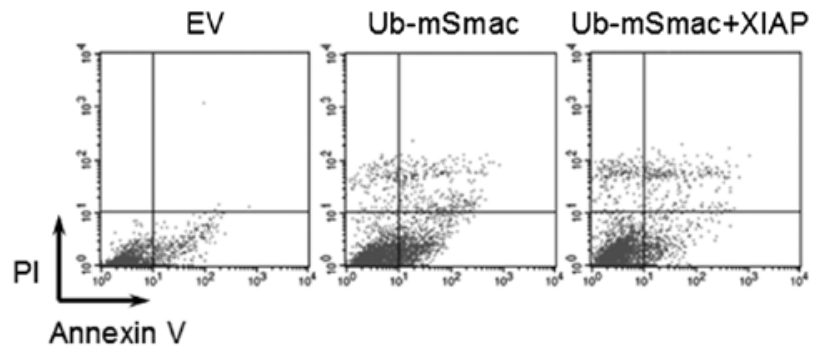

D

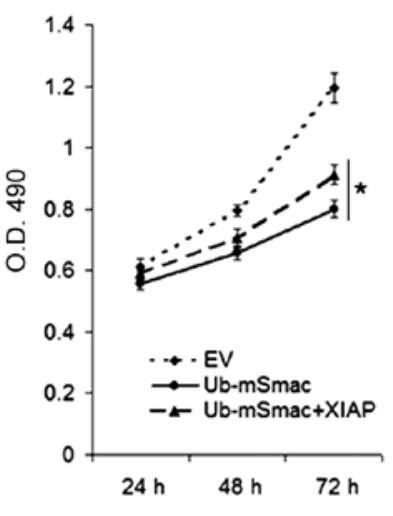

Figure 5. Mature Smac-induced H460 cell apoptosis is partially reverted by XIAP. (A) H460 cells successfully expressed Flag-Ub-mSmac and/or Myc-XIAP by transfection. (B) Cell apoptosis was measured by flow cytometry and (C) the apoptotic rates are provided as mean \pm SD. XIAP overexpression decreased the percentage of apoptotic cells in Ub-mSmac transfected H460 cells. (D) XIAP was found to enhance the viability of mSmac overexpressing H460 cells (MTT assay). (E) The activity of caspase- 3 in mSmac overexpressing H460 cells was decreased by Myc-XIAP transfection. Values are depicted as the mean \pm SD. ${ }^{*} \mathrm{P}<0.05$.

and implanted cells into nude mice via subcutaneous injection. Tumor growth curve demonstrated that mSmac slowed down H460 tumor growth in mice, while XIAP partially restored tumor growth for mSmac overexpressed H460 cells (Fig. 6A). After one month growth, the mice were sacrificed and the tumor tissues from the mice were detected by immunohistochemistry. As expected, the expression of mSmac was downregulated by XIAP overexpression. Overexpression of mSmac inhibited Ki-67 expression, while XIAP reversed this inhibition by degrading mSmac. Taken together, these data indicate that mature Smac inhibited tumor cell proliferation, but this effect was partially reversed by XIAP (Fig. 6B and C).

\section{Discussion}

In renal cell carcinoma, breast, bladder cancer and other solid tumors, the expression of XIAP is higher with a later TMN stage, worse tumor differentiation and higher recurrence rate. The overall survival of patient with high XIAP expression is much shorter than those with low XIAP expression (15-17). In contrast, as a pro apoptotic factor, Smac expression is lower in cancer tissues than that in normal tissues in hepatocellular carcinoma or testicular germ tumors, and its high expression indicates a good prognosis $(18,19)$. However, the level of Smac varies in different tumors. For example, in cervical cancer, the expression of Smac in cancer tissue is higher than in normal cervical tissue, especially in mRNA level (23). In this study, we found that the mRNA level of XIAP in lung cancer tissues was significantly higher than that in the corresponding normal tissues, while the mRNA level of Smac had no significant difference between normal and cancer tissues. However, the protein of XIAP in lung cancer tissue was significantly higher than that in the normal tissue, while the protein of Smac was significantly higher than that in normal tissue. Smac and XIAP were negatively correlated in protein levels but not in mRNA levels. It was possibly because the degradation of Smac by XIAP was at the level of protein.

Overexpression of full length of Smac increases chemo- or radiotherapy-induced cell apoptosis in lung, breast, ovarian cancer (24-27). In our previous studies, we found overexpression of Smac increased cisplatin and indomethacin-induced apoptosis because Smac released from mitochrondria into cytosol under the treatment of the drug, and then performed its function in lung cancer and esophageal cancer $(28,29)$. In this study, fSmac was not degraded by XIAP, and it did not promote apoptosis, whereas mSmac could be degraded by XIAP and induced apoptosis. It was because fSmac was localized in mitochondria, while mSmac was localized in the cytoplasm. In addition, Smac promoted apoptosis only if it was located in the cytoplasm, similarly, it bound to and degraded by XIAP only in the cytoplasm. The Ub and mature Smac fusion protein, which was the expressed production of the Flag-UbmSmac plasmid, can directly localized in the cytoplasm (30). It was easier to distinguish the speed of Smac degradation in the presence from absence of XIAP by using Flag-Ub-mSmac and Myc-XIAP plasmids. 
A

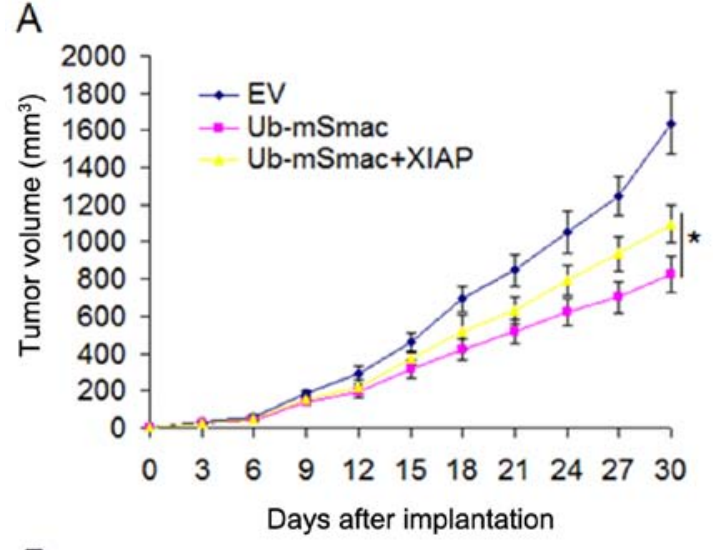

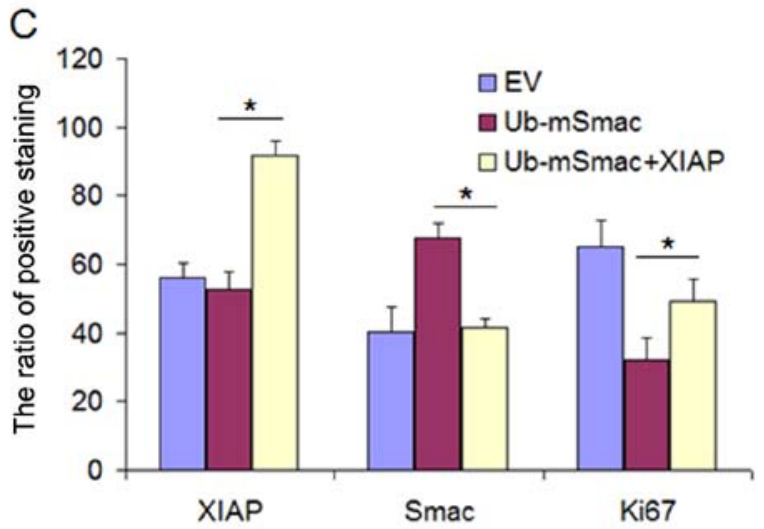

Ub-mSmac
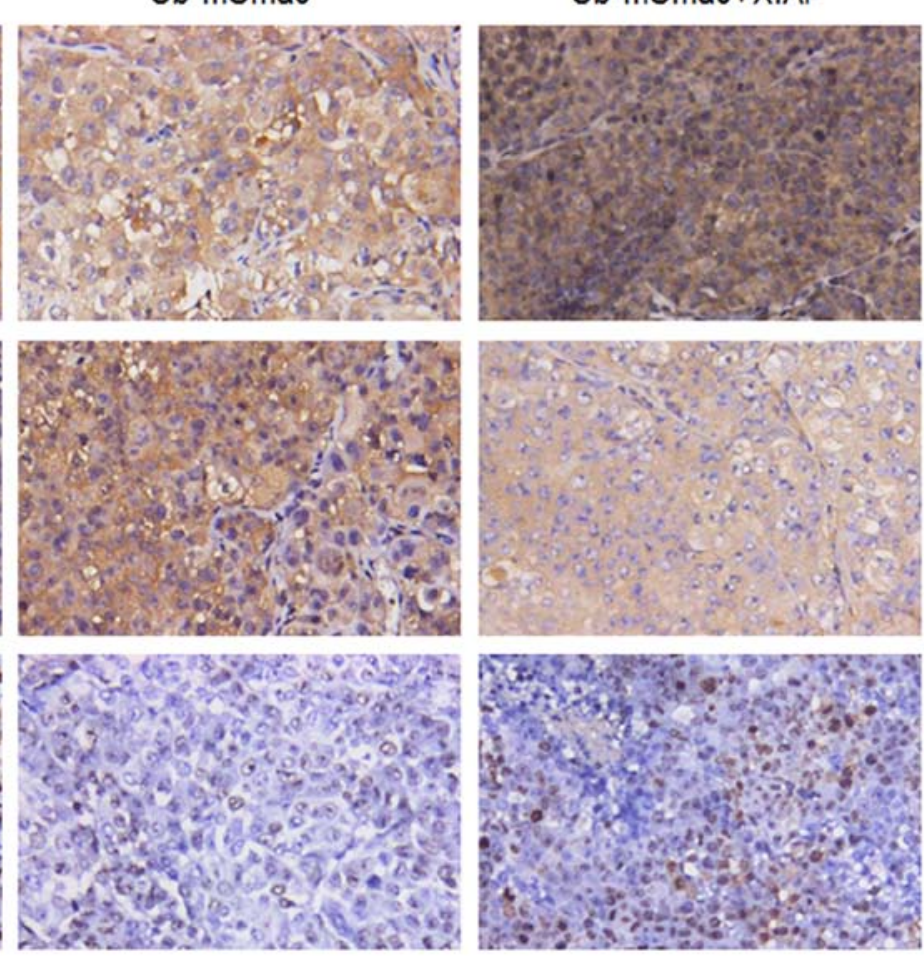

Figure 6. XIAP partially abolishes the mSmac inhibition of tumor growth. (A) Control H460 cells, Ub-mSmac overexpressed H460 cells and Ub-mSmac/ Myc-XIAP co-expressing H460 cells, respectively, were implanted into nude mice via subcutaneous injection. Tumor nodules were measured using a caliper at different times after implantation. (B) Tumor nodules were subjected to immunohistochemical staining for XIAP, Smac and Ki-67. (C) The percentage of $\mathrm{XIAP}, \mathrm{Smac}$ and $\mathrm{Ki}-67$ positive cells in tumors were depicted by quantitative analysis. Values are depicted as the mean $\pm \mathrm{SD}$. * $\mathrm{P}<0.05$.

IAPs play the role of E3 ligase by their RING domains, which are closely related to their self-ubiquitination $(3,31)$. Mature Smac in the cytoplasm participates in the self-ubiquitination of the IAP family members. Some studies reported that IAP proteins, such as XIAP, c-IAP1, c-IAP2 and Apollon, degraded Smac through the ubiquitin pathway (32-34), while others reported that Smac promoted the degradation of survivin, cIAP1, cIAP2, but not XIAP and livin $(35,36)$. To clarify, in this study, we found that mature Smac in the cytoplasm combined with XIAP, which accelerated mature Smac ubiquitination. However, the expression of XIAP was relatively stable in the half-life experiment, even in the cells co-transfected with Smac. It is highly possible that Smac promoted IAP self-ubiquitination, at the same time, IAP transfered ubiquitin to Smac by its E3 ubiquitin ligase and degraded Smac. Therefore, both of them were degraded faster after combination. But different IAP family members had different stability. XIAP is not easy to degrad, while c-IAP1, c-IAP2 are relatively unstable. Therefore, previous studies demonstrated that XIAP, c-IAP1 and c-IAP2 degraded Smac, while Smac degraded cIAP1, and c-IAP2, but not XIAP.

In the present study, we found that the mSmac in the cytoplasm increased apoptosis, and XIAP inhibited mSmac induced apoptosis by degradation of mSmac. It was clear that mSmac was unstable in the cell cytoplasm because it was easily degraded by XIAP or other IAP family members. Therefore, the function of endogenous mSmac is limited. Ala-Val-Pro-Ile, a four N-terminal residue of Smac, competed with caspases for binding to the BIR domain of IAP proteins, sequentially releasing caspases and causing cell apoptosis. A novel drug, Smac mimetics, was designed to treat cancer by mimicking this residue. Preclinical studies showed that Smac mimetics 
activated caspases and degraded cIAP1,2 to induce apoptosis. Moreover, Smac mimetics displayed their anticancer effectiveness in phase I and II clinical trials. Further study of this drug shows promising future in the field of apoptosis-related drug development.

\section{Acknowledgements}

The present study was supported by the National Natural Science Foundation of China, approved ID no. 81402506.

\section{References}

1. Evan GI and Vousden KH: Proliferation, cell cycle and apoptosis in cancer. Nature 411: 342-348, 2001.

2. Cotter TG: Apoptosis and cancer: The genesis of a research field. Nat Rev Cancer 9: 501-507, 2009.

3. Fulda S and Vucic D: Targeting IAP proteins for therapeutic intervention in cancer. Nat Rev Drug Discov 11: 109-124, 2012.

4. Schimmer AD, Dalili S, Batey RA and Riedl SJ: Targeting XIAP for the treatment of malignancy. Cell Death Differ 13: 179-188, 2006.

5. Kaufmann T, Strasser A and Jost PJ: Fas death receptor signalling: Roles of Bid and XIAP. Cell Death Differ 19: 42-50, 2012.

6. Van den Abeele P and Bertrand MJ: The role of the IAP E3 ubiquitin ligases in regulating pattern-recognition receptor signalling. Nat Rev Immunol 12: 833-844, 2012.

7. Qin S, Yang C, Li S, Xu C, Zhao Y and Ren H: Smac: Its role in apoptosis induction and use in lung cancer diagnosis and treatment. Cancer Lett 318: 9-13, 2012.

8. Morizane Y, Honda R, Fukami K and Yasuda H: X-linked inhibitor of apoptosis functions as ubiquitin ligase toward mature caspase-9 and cytosolic Smac/DIABLO. J Biochem 137: $125-132,2005$.

9. Flanagan L, Sebastià J, Tuffy LP, Spring A, Lichawska A, Devocelle M, Prehn JH and Rehm M: XIAP impairs Smac release from the mitochondria during apoptosis. Cell Death Dis 1: e 49, 2010

10. Li S, Sun J, Yang J, Zhang L, Wang L, Wang X and Guo Z: XIAP expression is associated with pancreatic carcinoma outcome. Mol Clin Oncol 1: 305-308, 2013.

11. Yi XP, Han T, Li YX, Long XY and Li WZ: Simultaneous silencing of XIAP and survivin causes partial mesenchymalepithelial transition of human pancreatic cancer cells via the PTEN/PI3K/Akt pathway. Mol Med Rep 12: 601-608, 2015.

12. Zhao YC, Wang Y, Ni XJ, Li Y, Wang XM, Zhu YY and Luo CY: Clinical significance of Smac and survivin expression in breast cancer patients treated with anthracycline-based neoadjuvant chemotherapy. Mol Med Rep 9: 614-620, 2014.

13. Shintani M, Sangawa A, Yamao N and Kamoshida S: Smac/ DIABLO expression in human gastrointestinal carcinoma: Association with clinicopathological parameters and survivin expression. Oncol Lett 8: 2581-2586, 2014.

14. Qu Y, Xia P, Zhang S, Pan S and Zhao J: Silencing XIAP suppresses osteosarcoma cell growth, and enhances the sensitivity of osteosarcoma cells to doxorubicin and cisplatin. Oncol Rep 33: 1177-1184, 2015.

15. Li M, Song T, Yin ZF and Na YQ: XIAP as a prognostic marker of early recurrence of nonmuscular invasive bladder cancer. Chin Med J (Engl) 120: 469-473, 2007.

16. Mizutani Y, Nakanishi H, Li YN, Matsubara H, Yamamoto K, Sato N, Shiraishi T, Nakamura T, Mikami K, Okihara K, et al: Overexpression of XIAP expression in renal cell carcinoma predicts a worse prognosis. Int J Oncol 30: 919-925, 2007.

17. Zhang Y, Zhu J, Tang Y, Li F, Zhou H, Peng B, Zhou C and Fu R: X-linked inhibitor of apoptosis positive nuclear labeling: A new independent prognostic biomarker of breast invasive ductal carcinoma. Diagn Pathol 6: 49, 2011.

18. Bao ST, Gui SQ and Lin MS: Relationship between expression of Smac and Survivin and apoptosis of primary hepatocellular carcinoma. Hepatobiliary Pancreat Dis Int 5: 580-583, 2006.
19. Kempkensteffen C, Jäger T, Bub J, Weikert S, Hinz S, Christoph F, Krause H, Schostak M, Miller K and Schrader M: The equilibrium of XIAP and Smac/DIABLO expression is gradually deranged during the development and progression of testicular germ cell tumours. Int J Androl 30: 476-483, 2007.

20. Yan Y, Mahotka C, Heikaus S, Shibata T, Wethkamp N, Liebmann J, Suschek CV, Guo Y, Gabbert HE, Gerharz CD, et al: Disturbed balance of expression between XIAP and Smac/ DIABLO during tumour progression in renal cell carcinomas. $\mathrm{Br}$ J Cancer 91: 1349-1357, 2004.

21. Shibata T, Mahotka C, Wethkamp N, Heikaus S, Gabbert HE and Ramp U: Disturbed expression of the apoptosis regulators XIAP, $\mathrm{XAF} 1$, and Smac/DIABLO in gastric adenocarcinomas. Diagn Mol Pathol 16: 1-8, 2007.

22. Qin S, Xu C, Li S, Wang X, Sun X, Wang P, Zhang B and Ren H: Hyperthermia induces apoptosis by targeting Survivin in esophageal cancer. Oncol Rep 34: 2656-2664, 2015.

23. Arellano-Llamas A, Garcia FJ, Perez D, Cantu D, Espinosa M, De la Garza JG, Maldonado V and Melendez-Zajgla J: High Smac/DIABLO expression is associated with early local recurrence of cervical cancer. BMC Cancer 6: 256, 2006.

24. Fandy TE, Shankar S and Srivastava RK: Smac/DIABLO enhances the therapeutic potential of chemotherapeutic drugs and irradiation, and sensitizes TRAIL-resistant breast cancer cells. Mol Cancer 7: 60, 2008.

25. McNeish IA, Bell S, McKay T, Tenev T, Marani $M$ and Lemoine NR: Expression of Smac/DIABLO in ovarian carcinoma cells induces apoptosis via a caspase-9-mediated pathway. Exp Cell Res 286: 186-198, 2003.

26. Maas C, Verbrugge I, de Vries E, Savich G, van de Kooij LW, Tait SW and Borst J: Smac/DIABLO release from mitochondria and XIAP inhibition are essential to limit clonogenicity of Type I tumor cells after TRAIL receptor stimulation. Cell Death Differ 17: 1613-1623, 2010.

27. Mao HL, Liu PS, Zheng JF, Zhang PH, Zhou LG, Xin G and Liu C: Transfection of Smac/DIABLO sensitizes drug-resistant tumor cells to TRAIL or paclitaxel-induced apoptosis in vitro. Pharmacol Res 56: 483-492, 2007.

28. Qin S, Yang C, Wang X, Xu C, Li S, Zhang B and Ren H: Overexpression of Smac promotes Cisplatin-induced apoptosis by activating caspase- 3 and caspase- 9 in lung cancer A549 cells. Cancer Biother Radiopharm 28: 177-182, 2013.

29. Qin S, Xu C, Li S, Yang C, Sun X, Wang X, Tang SC and Ren H: Indomethacin induces apoptosis in the EC109 esophageal cancer cell line by releasing second mitochondria-derived activator of caspase and activating caspase-3. Mol Med Rep 11: 4694-4700, 2015.

30. Hunter AM, Kottachchi D, Lewis J, Duckett CS, Korneluk RG and Liston P: A novel ubiquitin fusion system bypasses the mitochondria and generates biologically active Smac/DIABLO. J Biol Chem 278: 7494-7499, 2003.

31. Vucic D, Dixit VM and Wertz IE: Ubiquitylation in apoptosis: A post-translational modification at the edge of life and death. Nat Rev Mol Cell Biol 12: 439-452, 2011.

32. Hu S and Yang X: Cellular inhibitor of apoptosis 1 and 2 are ubiquitin ligases for the apoptosis inducer Smac/DIABLO. J Biol Chem 278: 10055-10060, 2003.

33. Hao Y, Sekine K, Kawabata A, Nakamura H, Ishioka T, Ohata H, Katayama R, Hashimoto C, Zhang X, Noda T, et al: Apollon ubiquitinates SMAC and caspase-9, and has an essential cytoprotection function. Nat Cell Biol 6: 849-860, 2004.

34. MacFarlane M, Merrison W, Bratton SB and Cohen GM: Proteasome-mediated degradation of Smac during apoptosis: XIAP promotes Smac ubiquitination in vitro. J Biol Chem 277: 36611-36616, 2002.

35. McNeish IA, Lopes R, Bell SJ, McKay TR, Fernandez M, Lockley M, Wheatley SP and Lemoine NR: Survivin interacts with Smac/DIABLO in ovarian carcinoma cells but is redundant in Smac-mediated apoptosis. Exp Cell Res 302: 69-82, 2005.

36. Yang QH and Du C: Smac/DIABLO selectively reduces the levels of c-IAP1 and c-IAP2 but not that of XIAP and livin in HeLa cells. J Biol Chem 279: 16963-16970, 2004. 